\title{
A RECONSIDERATION OF HUA'S INEQUALITY
}

HIROYUKI TAKAGI, TAKESHI MIURA, TADASHI KANZO, AND SIN-EI TAKAHASI

Received 11 July 2004

The inequality discovered by L. K. Hua in 1965 has been generalized in several directions. In this paper, we adopt a certain conjugate method to give a simple and fundamental inequality on two functions on a semigroup, which is the key to the proof of many generalizations of Hua's inequality.

\section{Introduction}

In 1965, L. K. Hua discovered the following inequality which plays an important role in number theory.

Theorem 1.1 (Hua's inequality [2]). If $\delta, \alpha>0$ and $x_{1}, \ldots, x_{n} \in \mathbb{R}$, then

$$
\left(\delta-\sum_{i=1}^{n} x_{i}\right)^{2}+\alpha \sum_{i=1}^{n} x_{i}^{2} \geq \frac{\alpha \delta^{2}}{\alpha+n}
$$

In (1.1), the equality holds if and only if $x_{1}=\cdots=x_{n}=\delta /(\alpha+n)$.

This inequality has been generalized as follows.

Theorem 1.2 (Wang's inequality [6]). If $p>1$, then

$$
\left|\delta-\sum_{i=1}^{n} x_{i}\right|^{p}+\alpha^{p-1} \sum_{i=1}^{n}\left|x_{i}\right|^{p} \geq\left(\frac{\alpha}{\alpha+n}\right)^{p-1} \delta^{p}
$$

for any $\delta, \alpha>0, x_{1}, \ldots, x_{n} \in \mathbb{R}$. If $0<p<1$, then the inequality sign in (1.2) is reversed, where $\delta, \alpha>0, x_{1}, \ldots, x_{n} \geq 0$ with $\sum_{i=1}^{n} x_{i} \leq \delta$. In both cases, the equality holds in (1.2) if and only if $x_{1}=\cdots=x_{n}=\delta /(\alpha+n)$. 
Theorem 1.3 (Dragomir-Yang inequalities [1]). Let $X$ be a real inner product space. Then

$$
\begin{gathered}
(\delta-\langle x, y\rangle)^{2}+\alpha\|x\|^{2} \geq \frac{\alpha \delta^{2}}{\alpha+\|y\|^{2}}, \\
\left\|y-\sum_{i=1}^{n} x_{i}\right\|^{2}+\alpha \sum_{i=1}^{n}\left\|x_{i}\right\|^{2} \geq \frac{\alpha}{\alpha+n}\|y\|^{2}
\end{gathered}
$$

for any $\delta, \alpha>0, x, y, x_{1}, \ldots, x_{n} \in X$. In (1.3), the equality holds if and only if $x=(\delta /(\alpha+$ $\left.\left.\|y\|^{2}\right)\right) y$. In (1.4), the equality holds if and only if $x_{1}=\cdots=x_{n}=(1 /(\alpha+n)) y$.

In [4], Pearce and Pečarić pointed out that these inequalities are consequences of Jensen's inequality. Moreover, Pečarić [5] showed the following three inequalities.

Theorem 1.4 (Pečarić's inequalities [5]). Let $X$ be a real inner product space. If $p, q>1$ and $1 / p+1 / q=1$, then

$$
|\delta-\langle x, y\rangle|^{p}+\alpha^{p-1}\|x\|^{p} \geq\left(\frac{\alpha}{\alpha+\|y\|^{q}}\right)^{p-1} \delta^{p}
$$

for any $\delta, \alpha>0, x, y \in X$. The equality holds in (1.5) if and only if $x=y=0$ or $x=$ $\left(\delta\|y\|^{q-2} /\left(\alpha+\|y\|^{q}\right)\right) y$. Moreover, if $f$ is a nondecreasing convex function on $[0, \infty)$, then

$$
\begin{gathered}
f(|\delta-\langle x, y\rangle|)+\frac{\|y\|}{\alpha} f(\alpha\|x\|) \geq \frac{\alpha+\|y\|}{\alpha} f\left(\frac{\alpha \delta}{\alpha+\|y\|}\right), \\
f\left(\left\|y-\sum_{i=1}^{n} x_{i}\right\|\right)+\frac{1}{\alpha} \sum_{i=1}^{n} f\left(\alpha\left\|x_{i}\right\|\right) \geq \frac{\alpha+n}{\alpha} f\left(\frac{\alpha\|y\|}{\alpha+n}\right)
\end{gathered}
$$

for any $\delta, \alpha>0, x, y, x_{1}, \ldots, x_{n} \in X$. When $f$ is strictly convex, the equality in (1.6) holds if and only if $y=0$ or $x=(\delta /(\|y\|(\alpha+\|y\|))) y$, and the equality in (1.7) holds if and only if $x_{1}=\cdots=x_{n}=(1 /(\alpha+n)) y$.

In this paper, we use a certain conjugate method to give a more extended formulation including the above inequalities.

\section{Results}

The main result of this paper is the following inequality.

Theorem 2.1. Let $(G,+)$ be a semigroup, and let $\varphi$ and $\psi$ be nonnegative functions on $G$. Suppose $\varphi$ is subadditive on $G$ and there is a positive constant $\lambda$ such that $\varphi(x) \leq \lambda \psi(x)$ for $x \in G$. If $f$ is a nondecreasing convex function on $[0, \infty)$, then

$$
f\left(\varphi\left(a_{0}\right)\right)+\lambda \sum_{i=1}^{n} f\left(\psi\left(a_{i}\right)\right) \geq(1+n \lambda) f\left(\frac{1}{1+n \lambda} \varphi\left(\sum_{i=0}^{n} a_{i}\right)\right)
$$


for any $a_{0}, a_{1}, \ldots, a_{n} \in G$. When $f$ is strictly convex, the equality holds in (2.1) if and only if

$$
\varphi\left(\sum_{i=0}^{n} a_{i}\right)=\sum_{i=0}^{n} \varphi\left(a_{i}\right), \quad \varphi\left(a_{i}\right)=\lambda \psi\left(a_{i}\right)(i=1, \ldots, n), \quad \varphi\left(a_{0}\right)=\psi\left(a_{1}\right)=\cdots=\psi\left(a_{n}\right) .
$$

The proof is elementary.

Proof. From the hypotheses of $\varphi$ and $\psi$, we have

$$
\varphi\left(\sum_{i=0}^{n} a_{i}\right) \leq \sum_{i=0}^{n} \varphi\left(a_{i}\right)=\varphi\left(a_{0}\right)+\sum_{i=1}^{n} \varphi\left(a_{i}\right) \leq \varphi\left(a_{0}\right)+\sum_{i=1}^{n} \lambda \psi\left(a_{i}\right) .
$$

Since $f$ is nondecreasing and convex, it follows that

$$
\begin{aligned}
f\left(\frac{1}{1+n \lambda} \varphi\left(\sum_{i=0}^{n} a_{i}\right)\right) & \leq f\left(\frac{1}{1+n \lambda}\left(\varphi\left(a_{0}\right)+\sum_{i=1}^{n} \lambda \psi\left(a_{i}\right)\right)\right) \\
& \leq \frac{1}{1+n \lambda} f\left(\varphi\left(a_{0}\right)\right)+\sum_{i=1}^{n} \frac{\lambda}{1+n \lambda} f\left(\psi\left(a_{i}\right)\right) .
\end{aligned}
$$

This gives (2.1). If $f$ is strictly convex, then $f$ is increasing, and so the equality occurs in (2.1) precisely when all the inequality signs in (2.3) and (2.4) become equality signs. Hence (2.2) is the condition for the equality in (2.1).

Remark 2.2. Condition (2.2) can be written as follows:

$$
\varphi\left(\sum_{i=0}^{n} a_{i}\right)=(1+n \lambda) \varphi\left(a_{0}\right), \quad \varphi\left(a_{0}\right)=\psi\left(a_{1}\right)=\cdots=\psi\left(a_{n}\right) .
$$

Theorem 2.1 has three useful corollaries.

Corollary 2.3. Let $(G,+)$ be a semigroup, and let $\varphi$ and $\psi$ be nonnegative functions on $G$. Suppose $\varphi$ is additive on $G$ and there is a positive constant $\lambda$ such that $\varphi(x)=\lambda \psi(x)$ for $x \in$ G. If $f$ is a convex function on $[0, \infty)$, then inequality (2.1) holds for any $a_{0}, a_{1}, \ldots, a_{n} \in G$. When $f$ is strictly convex, the equality holds in (2.1) if and only if $\varphi\left(a_{0}\right)=\psi\left(a_{1}\right)=\cdots=$ $\psi\left(a_{n}\right)$.

Proof. We can prove Corollary 2.3 similarly as in Theorem 2.1. Thus examine the proof of Theorem 2.1.

Corollary 2.4. Under the hypotheses of Theorem 2.1, inequality

$$
f(\varphi(a))+\lambda f(\psi(b)) \geq(1+\lambda) f\left(\frac{\varphi(a+b)}{1+\lambda}\right)
$$


holds for any $a, b \in G$. When $f$ is strictly convex, the equality holds in (2.6) if and only if

$$
\varphi(a+b)=\varphi(a)+\varphi(b), \quad \varphi(b)=\lambda \psi(b), \quad \varphi(a)=\psi(b) .
$$

Proof. Put $n=1, a_{0}=a$, and $a_{1}=b$ in Theorem 2.1.

Remark 2.5. Condition (2.7) is equivalent to

$$
\varphi(a+b)=(1+\lambda) \varphi(a), \quad \varphi(a)=\psi(b) .
$$

Remark 2.6. In Theorem 2.1 and Corollaries 2.3 and 2.4, if we replace the words "nondecreasing" and "convex" by "nonincreasing" and "concave", respectively, then the inequality signs in (2.1) and (2.6) are reversed. In fact, consider the function $-f$.

Corollary 2.7. Let $X$ be a real or complex normed space with dual $X^{*}$. If $p, q>1$ and $1 / p+1 / q=1$, then

$$
|\delta-h(x)|^{p}+\alpha^{p-1}\|x\|^{p} \geq\left(\frac{\alpha}{\alpha+\|h\|^{q}}\right)^{p-1} \delta^{p}
$$

for any $\delta, \alpha>0, x \in X, h \in X^{*}$. The equality holds in (2.9) if and only if $h(x)=\|h\|\|x\|$ and $\|x\|=\delta\|h\|^{q-1} /\left(\alpha+\|h\|^{q}\right)$ (and hence $h$ is a norm-attaining functional).

Proof. If $h$ is the zero functional on $X$, then (2.9) is trivial, and it becomes an equality precisely when $x=0$. So, assume that $h$ is nonzero. To prove (2.9), we use Corollary 2.4. Take $G=X$. Define $\varphi$ and $\psi$ by $\varphi(x)=|h(x)|$ and $\psi(x)=\alpha\|h\|^{1-q}\|x\|$ for any $x \in X$. Then $\varphi$ is subadditive on $X$ and $\varphi(x) \leq\|h\|\|x\|=\lambda \psi(x)$ for $x \in X$, where $\lambda=\|h\|^{q / \alpha}$. Moreover, put $f(t)=t^{p}$ for $t \geq 0$. Then $f$ is nondecreasing and convex on $[0, \infty)$. Now, apply Corollary 2.4. Then we have

$$
|h(a)|^{p}+\alpha^{p-1}\|b\|^{p} \geq\left(1+\frac{\|h\|^{q}}{\alpha}\right)^{1-p}|h(a+b)|^{p}
$$

for $a, b \in X$, and the equality holds if and only if

$$
|h(a+b)|=|h(a)|+|h(b)|, \quad|h(b)|=\|h\|\|b\|, \quad|h(a)|=\alpha\|h\|^{1-q}\|b\| .
$$

Now choose $y \in X$ so that $h(y)=\delta$, and replace $a$ and $b$ by $y-x$ and $x$, respectively. Then (2.10) implies (2.9), and the equality holds in (2.9) if and only if

$$
\begin{gathered}
\delta=|\delta-h(x)|+|h(x)|, \\
|h(x)|=\|h\|\|x\|, \\
|\delta-h(x)|=\alpha\|h\|^{1-q}\|x\| .
\end{gathered}
$$

From (2.12), we can easily see that $0 \leq h(x) \leq \delta$. Hence (2.13) and (2.14) become $h(x)=$ $\|h\|\|x\|$ and $\delta-h(x)=\alpha\|h\|^{1-q}\|x\|$. By deleting the term $h(x)$ from these equations, we obtain $\|x\|=\delta\|h\|^{q-1} /\left(\alpha+\|h\|^{q}\right)$. Consequently, we have

$$
h(x)=\|h\|\|x\|, \quad\|x\|=\frac{\delta\|h\|^{q-1}}{\alpha+\|h\|^{q}} .
$$


Also, the simple computation shows that (2.15) implies (2.12), (2.13), and (2.14). Hence (2.15) is the condition for the equality in (2.9). Thus we proved Corollary 2.7.

\section{Connections between inequalities}

Inequalities (1.1), (1.2), (1.3), (1.4), (1.5), (1.6), (1.7), (2.1), (2.6), and (2.9) are connected as in Figure 3.1 .

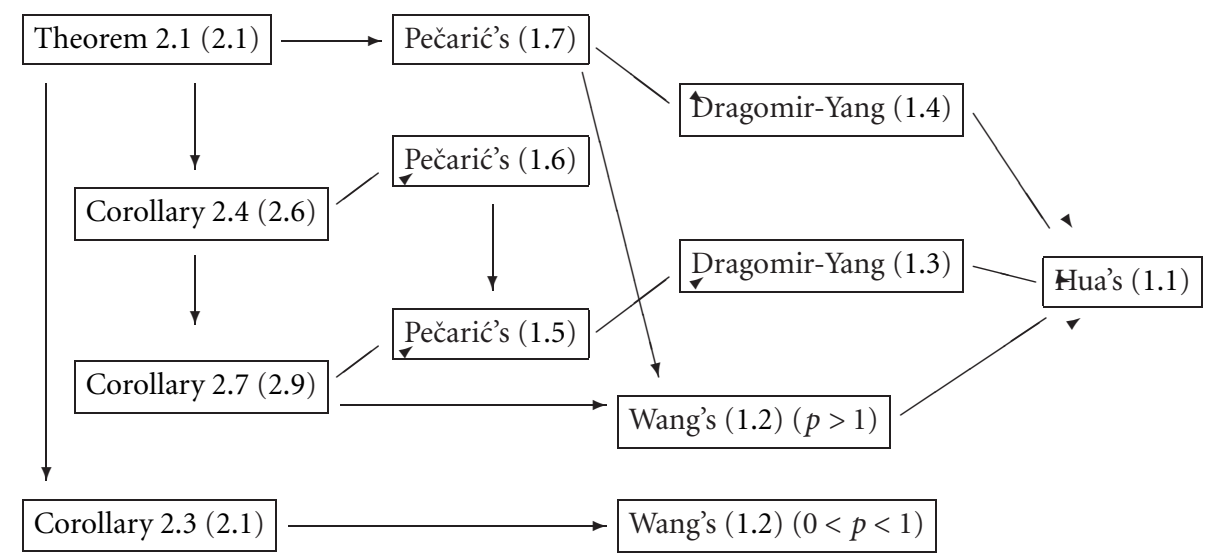

Figure 3.1

In what follows, we will explain the relations in Figure 3.1.

(1) Inequality (2.1) implies Pečarić's inequality (1.7). Take $G=X$ and put $\varphi(x)=\|x\|$ and $\psi(x)=\alpha\|x\|$ for $x \in X$. Then $\varphi$ is subadditive on $X$ and $\varphi(x)=\lambda \psi(x)$ for $x \in X$, where $\lambda=1 / \alpha$. Apply Theorem 2.1 with $a_{0}=y-\sum_{i=1}^{n} x_{i}$ and $a_{i}=x_{i}$ for $i=1, \ldots, n$. Then (2.1) yields (1.7), and (2.2) becomes

$$
\begin{gathered}
\|y\|=\left\|y-\sum_{i=1}^{n} x_{i}\right\|+\sum_{i=1}^{n}\left\|x_{i}\right\|, \quad\left\|x_{i}\right\|=\frac{1}{\alpha} \alpha\left\|x_{i}\right\|(i=1, \ldots, n), \\
\left\|y-\sum_{i=1}^{n} x_{i}\right\|=\alpha\left\|x_{1}\right\|=\cdots=\alpha\left\|x_{n}\right\|,
\end{gathered}
$$

which is the condition for the equality in (1.7). In (3.1), the second equation always holds. The first equation says that equality holds in the triangle inequality, and we can find a unit element $z$ in $X$ such that

$$
y-\sum_{i=1}^{n} x_{i}=\left\|y-\sum_{i=1}^{n} x_{i}\right\| z, \quad x_{1}=\left\|x_{1}\right\| z, \ldots, x_{n}=\left\|x_{n}\right\| z .
$$


Combining with the third equation, we have $y-\sum_{i=1}^{n} x_{i}=\alpha\left\|x_{1}\right\| z=\alpha x_{1}$ and $x_{1}=\cdots=$ $x_{n}$. Hence

$$
x_{1}=\cdots=x_{n}=\frac{1}{\alpha+n} y .
$$

Meanwhile, it is obvious that (3.3) implies (3.1). Thus (3.3) is the condition for the equality in (1.7).

Remark 3.1. In the above observation, we use the condition for equality in the triangle inequality in an inner product space: $\left\|x_{1}+\cdots+x_{n}\right\|=\left\|x_{1}\right\|+\cdots+\left\|x_{n}\right\|$ if and only if there is a unit element $z$ such that $x_{i}=\left\|x_{i}\right\| z$ for $i=1, \ldots, n$. The condition in a Banach space is investigated in [3].

(2) Inequality (2.6) implies Pečarić's inequality (1.6). If $y=0$, then (1.6) holds clearly as equality. Fix a nonzero $y \in X$. Take $G=X$. Put $\varphi(x)=|\langle x, y\rangle|$ and $\psi(x)=\alpha\|x\|$ for any $x \in X$. Then $\varphi$ is subadditive on $X$ and $\varphi(x) \leq\|x\|\|y\|=\lambda \psi(x)$ for $x \in X$, where $\lambda=\|y\| / \alpha$. Choose $z \in X$ so that $\delta=\langle z, y\rangle$. Applying Corollary 2.4 with $a=z-x$ and $b=x$, we obtain (1.6) from (2.6), and the condition for equality in (1.6) is

$$
\delta=|\delta-\langle x, y\rangle|+|\langle x, y\rangle|, \quad|\langle x, y\rangle|=\|x\|\|y\|, \quad|\delta-\langle x, y\rangle|=\alpha\|x\| .
$$

In (3.4), the first equation tells us that $0 \leq\langle x, y\rangle \leq \delta$, and so the second and third equations become $\langle x, y\rangle=\|x\|\|y\|$ and $\delta-\langle x, y\rangle=\alpha\|x\|$. Deleting the term $\langle x, y\rangle$ from these equations, we get $\|x\|=\delta /(\alpha+\|y\|)$. Moreover, since the equality holds in the CauchySchwartz inequality $\langle x, y\rangle \leq\|x\|\|y\|$, it follows that $x=(\|x\| /\|y\|) y$. Hence

$$
x=\frac{\delta}{\|y\|(\alpha+\|y\|)} y .
$$

Also, we can easily check that (3.5) implies (3.4). Thus, in case $y \neq 0,(3.5)$ is the condition for the equality in (1.6).

(3) Inequality (2.9) implies Pečarić's inequality (1.5). Fix $y \in X$ and put $h(x)=\langle x, y\rangle$ for any $x \in X$. Then $h \in X^{*}$ and $\|h\|=\|y\|$. Hence (1.5) follows immediately from (2.9). Moreover, the equality holds in (1.5) if and only if

$$
\langle x, y\rangle=\|x\|\|y\|, \quad\|x\|=\frac{\delta\|y\|^{q-1}}{\alpha+\|y\|^{q}} .
$$

From the first equation, it follows that $\|y\| x=\|x\| y$. Combining with the second equation, we see that $\|y\| x=\left(\delta\|y\|^{q-1} /\left(\alpha+\|y\|^{q}\right)\right) y$. Thus we obtain

$$
x=y=0 \quad \text { or } \quad x=\frac{\delta\|y\|^{q-2}}{\alpha+\|y\|^{q}} y .
$$

Conversely (3.7) implies (3.6). Hence (3.7) is the condition for the equality in (1.5).

(4) Inequality (2.9) implies the case $p>1$ of Wang's inequality (1.2). Suppose $p>1$ and $1 / p+1 / q=1$. Let $X$ be the space $\mathbb{R}^{n}$ with norm $\left\|\left(x_{1}, \ldots, x_{n}\right)\right\|=\left(\sum_{i=1}^{n}\left|x_{i}\right|^{p}\right)^{1 / p}$. We 
take $h \in X^{*}$ to be the functional corresponding to $(1, \ldots, 1) \in \mathbb{R}^{n}$, that is,

$$
h\left(\left(x_{1}, \ldots, x_{n}\right)\right)=\sum_{i=1}^{n} x_{i}
$$

for any $\left(x_{1}, \ldots, x_{n}\right) \in \mathbb{R}^{n}=X$. As is well known, $\|h\|=\left(\sum_{i=1}^{n} 1^{q}\right)^{1 / q}=n^{1 / q}$. Now we apply Corollary 2.7. Then (1.2) follows directly from (2.9). Moreover, the equality holds in (1.2) if and only if

$$
\sum_{i=1}^{n} x_{i}=n^{1 / q}\left(\sum_{i=1}^{n}\left|x_{i}\right|^{p}\right)^{1 / p}, \quad\left(\sum_{i=1}^{n}\left|x_{i}\right|^{p}\right)^{1 / p}=\frac{\delta n^{1 / p}}{\alpha+n}
$$

By the first equation and Hölder's inequality, we have

$$
n^{1 / q}\left(\sum_{i=1}^{n}\left|x_{i}\right|^{p}\right)^{1 / p}=\sum_{i=1}^{n} x_{i} \leq \sum_{i=1}^{n}\left|x_{i}\right| \leq n^{1 / q}\left(\sum_{i=1}^{n}\left|x_{i}\right|^{p}\right)^{1 / p},
$$

and the two inequality signs become equal signs. We recall the condition for equality in Hölder's inequality and see that $\left|x_{1}\right|=\cdots=\left|x_{n}\right|$. In addition, we have $\sum_{i=1}^{n} x_{i}=$ $\sum_{i=1}^{n}\left|x_{i}\right|$, and so $x_{1}=\cdots=x_{n} \geq 0$. Together with the second equation in (3.9), we have

$$
x_{1}=\cdots=x_{n}=\frac{\delta}{\alpha+n} \text {. }
$$

Also, we easily check that (3.11) implies (3.9). Consequently, (3.11) is the condition for the equality in (1.2).

(5) Corollary 2.3 (2.1) implies the case $0<p<1$ of Wang's inequality (1.2). As G, take the additive semigroup $\mathbb{R}^{+}$of all nonnegative real numbers. Put $\varphi(x)=x$ and $\psi(x)=\alpha x$ for $x \in \mathbb{R}^{+}$. Then $\varphi$ is additive on $\mathbb{R}^{+}$and $\varphi(x)=\lambda \psi(x)$ for $x \in \mathbb{R}^{+}$, where $\lambda=1 / \alpha$. Suppose $0<p<1$ and put $f(t)=t^{p}$ for $t \geq 0$. Then $f$ is concave on $[0, \infty)$. Now apply Corollary 2.3 and Remark 2.6 with $a_{0}=\delta-\sum_{i=1}^{n} x_{i}$ and $a_{i}=x_{i}$ for $i=1, \ldots, n$, where $x_{1}, \ldots, x_{n} \geq 0$ and $\sum_{i=1}^{n} x_{i} \leq \delta$. Then (2.1) gives the reverse inequality of (1.2). Also, the condition for the equality in it is

$$
\delta-\sum_{i=1}^{n} x_{i}=\alpha x_{1}=\cdots=\alpha x_{n}
$$

which is equivalent to $x_{1}=\cdots=x_{n}=\delta /(\alpha+n)$.

Remark 3.2. The reverse inequality of (1.2) in case $0<p<1$ is false for all $x_{1}, \ldots, x_{n} \geq 0$. A counterexample is $\delta=\alpha=1, n=1, x_{1}=2\left(x_{1}>\delta\right)$. 
(6) In Theorem 1.4, (1.6) implies (1.5). If $y=0$, then (1.5) is clearly true, and it becomes an equality exactly when $x=0$. In case $y \neq 0$, put $f(t)=t^{p}(t \geq 0)$ and replace $\alpha$ by $\alpha /\|y\|^{q-1}$ in (1.6).

(7) Pečarić's inequality (1.7) implies the case $p>1$ of Wang's inequality (1.2). In (1.7), put $X=\mathbb{R}, y=\delta$, and $f(t)=t^{p}(t \geq 0)$.

(8) Pečarić's inequality (1.7) implies Dragomir-Yang inequality (1.4). In (1.7), put $f(t)=$ $t^{2}(t \geq 0)$.

(9) Pečarićs inequality (1.5) implies Dragomir-Yang inequality (1.3). In (1.5), put $p=$ $q=2$.

Remark 3.3. Pečarić's inequality (1.5) plus Hölder's inequality implies the case $p>1$ of Wang's inequality (1.2). In (1.5), put $X=\mathbb{R}, x=\sum_{i=1}^{n} x_{i}, y=1$, and replace $\alpha$ by $\alpha / n$. Then we have

$$
\left|\delta-\sum_{i=1}^{n} x_{i}\right|^{p}+\left(\frac{\alpha}{n}\right)^{p-1}\left|\sum_{i=1}^{n} x_{i}\right|^{p} \geq\left(\frac{\alpha}{\alpha+n}\right)^{p-1} \delta^{p} .
$$

Meanwhile, by the triangle inequality and Hölder's inequality, we have

$$
\left|\sum_{i=1}^{n} x_{i}\right| \leq \sum_{i=1}^{n}\left|x_{i}\right| \leq n^{1 / q}\left(\sum_{i=1}^{n}\left|x_{i}\right|^{p}\right)^{1 / p}
$$

or

$$
\left|\sum_{i=1}^{n} x_{i}\right|^{p} \leq n^{p-1} \sum_{i=1}^{n}\left|x_{i}\right|^{p} .
$$

These inequalities imply (1.2). The condition for equality in (1.2) is obtained by using the argument in (4).

(10) Dragomir-Yang inequality (1.4) implies Hua's inequality (1.1). In (1.4), put $X=\mathbb{R}$ and $y=\delta$.

(11) Dragomir-Yang inequality (1.3) implies Hua's inequality (1.1). In (1.3), let $X$ be the $n$-dimensional Euclidean space $\mathbb{R}^{n}$ and take $x=\left(x_{1}, \ldots, x_{n}\right), y=(1, \ldots, 1) \in \mathbb{R}^{n}$.

(12) Wang's inequality (1.2) implies Hua's inequality (1.1). In (1.2), put $p=2$.

\section{Acknowledgment}

The second and fourth authors are partially supported by the Grant-in-Aid for Scientific Research, Japan Society for the Promotion of Science.

\section{References}

[1] S. S. Dragomir and G.-S. Yang, On Hua's inequality in real inner product spaces, Tamkang J. Math. 27 (1996), no. 3, 227-232.

[2] L. K. Hua, Additive Theory of Prime Numbers, Translations of Mathematical Monographs, vol. 13, American Mathematical Society, Rhode Island, 1965.

[3] R. Nakamoto and S.-E. Takahasi, Norm equality condition in triangular inequality, Sci. Math. Jpn. 55 (2002), no. 3, 463-466. 
[4] C. E. M. Pearce and J. E. Pečarić, A remark on the Lo-Keng Hua inequality, J. Math. Anal. Appl. 188 (1994), no. 2, 700-702.

[5] J. Pečarić, On Hua's inequality in real inner product spaces, Tamkang J. Math. 33 (2002), no. 3, 265-268.

[6] C.-L. Wang, Lo-Keng Hua inequality and dynamic programming, J. Math. Anal. Appl. 166 (1992), no. 2, 345-350.

Hiroyuki Takagi: Department of Mathematical Sciences, Faculty of Science, Shinshu University, Matsumoto 390-8621, Japan

E-mail address: takagi@math.shinshu-u.ac.jp

Takeshi Miura: Department of Basic Technology, Applied Mathematics and Physics, Yamagata University, Yonezawa 992-8510, Japan

E-mail address: miura@yz.yamagata-u.ac.jp

Tadashi Kanzo: College of Engineering, Kanto Gakuin University, Yokohama 236-8501, Japan

E-mail address: kanzo@kanto-gakuin.ac.jp

Sin-Ei Takahasi: Department of Basic Technology, Applied Mathematics and Physics, Yamagata University, Yonezawa 992-8510, Japan

E-mail address: sin-ei@emperor.yz.yamagata-u.ac.jp 